\begin{tabular}{lccr} 
A R C H I V E & O F & M E C H A N I C A L & E N G I N E E R I N G \\
\hline VOL. LX & 2013 & Number 4 \\
10.2478/meceng-2013-0034 & \\
Key words: robot, arm rehabilitation, EMG, exoskeleton, upper-extremity rehabilitation, upper-limb rehabilitation
\end{tabular}

ARTUR GMEREK *

\title{
MECHANICAL AND HARDWARE ARCHITECTURE OF THE SEMI-EXOSKELETON ARM REHABILITATION ROBOT
}

This paper deals with mechanical and hardware design of a robot, used for the rehabilitation of upper extremities. It has been called ARR-1 (Arm Rehabilitation Robot). The robot has a semi-exoskeleton structure. This means that some parts of the robot fit closely to the human arm (an orthosis), but the weight of the construction does not load patient's body. The device is used for the whole arm rehabilitation, but active joints are only situated in glenohumeral and elbow joints. The robot is electrically actuated.

\section{Introduction}

Millions of people around the world annually suffer from different articulation lesions, neurological impairments and cerebral damages. These people should be rehabilitated. The nervous system can reorganize after injury only by doing specific exercises. Also the quality of life of stroke patients can be easily improved. Hemiplegia or hemiparesis of the upper extremities can be reduced and rehabilitation can also decrease abnormal muscle coactivation and reorganize cortex. In patients with muscular dystrophy symptoms, rehabilitation can improve movement coordination and muscular strength.

Rehabilitation treatment is usually long, arduous and costly. It is a big branch of public health system, whose role is to instantly intensify the process of treatment, mainly because of the ageing of the society.

Robots can increase efficiency and effectiveness of therapy. Roboticassisted rehabilitation can reduce costs and lead to new effective therapeutic procedures. Automatic rehabilitation will also reduce the number of therapists required per patient.

* Lodz University of Technology, Institute of Automatic Control, 90-924 Lodz, Poland; E-mail: artur.gmerek@p.lodz.pl 
This work describes a mechanical and hardware design of the robot used for upper extremity rehabilitation. Inspiration for its construction was taken from studies on the existing robot of similar type and biomechanics of the human arm [1]. The robot was designed in the way that allows it to perform the widest possible type of rehabilitations. It can be used for repetitive therapy of people with cerebral damage, as well as for exercises for patients with impaired arms. The construction is a prototype structure based on the modular architecture approach. There is no need for dismantling the whole robot in order to exchange some parts for new ones. The control system is not limited by the construction of the robot. In the future, it will be, for example, possible to implement impedance/admittance control mode, as well as shakes therapy.

\section{Related Work}

Major development of robotic rehabilitation began almost 20 years ago, when Hogan et al. constructed a simple rehabilitation robot - MIT-MANUS [2] and developed a new type of algorithm, used for training [3]. The main disadvantage of the pioneering robots were their non extensive range of motion (ROM), planar movements and rehabilitation limited only to selected exercises. In the new constructions, these limitations have been reduced. Nowadays designers create many different robots and algorithms much more advanced then the pioneering systems. In scientific literature and databases of patents, there are hundreds of articles and descriptions of new robotic rehabilitation systems. Some of them have been already tested in clinical facilities and hospitals, as well as there are some commercial versions for sell. The most advanced are the constructions in which the upper extremity is connected to the robot in more than one place (usually in upper arm and forearm). This type of solution causes that the position of the upper arm to the forearm can be strictly controlled. In the following retrospect, mainly these structures are taken into account.

Engineers use many types of actuators. Each of them has advantages, but also disadvantages. For example pneumatic or hydraulic actuators are less rigid than electric actuators. Nonetheless, the first ones are more difficultly adoptable to the construction, more complicated to be controlled with advanced algorithms and need additional generally costly devices for compressed air preparation. In spite of that, there are a few interesting constructions, which have been tested on patients.

Pneu-WREX has 4 degrees of freedom (DOF) [4, 5]. It is equipped with pneumatic actuators attached to a lightweight exoskeleton. It includes movements like shoulder flexion/extension, forward/backward clavicle rota- 
tion, horizontal abduction/adduction and elbow flexion/extension. It moves in wide ROM in 3D space, and there have been already developed many types of advanced control algorithm.

Another interesting example of pneumatic actuated robots is iPAM [6]. It is a dual-arm robot system. One arm is connected to an upper arm of a patient and another to a forearm. It has 6 DOF and is able to draw patient's hand in 3D Cartesian space.

Mistry et al. developed a hydraulically actuated exoskeleton arm with anthropomorphic design [7]. The robot has 7 active DOF. Trajectory and torques generated by human arm can be reflected by the apparatus.

Some constructions use pneumatic muscle actuators. A good example of this type of robots is Salford Rehabilitation Exoskeleton (SRE) [8]. It is an upper arm assistive exoskeleton with light framework. It has 7 active DOF. Three of them fall on shoulder and 2 on elbow joints. Besides, they enable flexion/extension and pronation/supination of the forearm. The muscles are situated on the base behind the robot. Joints are moved via cables.

RUPERT IV (Robotic Upper Extremity Repetitive Trainer) is a 5 DOF pneumatic muscle exoskeleton $[9,10]$. The whole robot loads on patient shoulders and back. For this reason, it is limited to selected exercises only. Device is wearable and light.

Some interesting experiments have also been conducted by Umemura and his colleagues [11]. They developed a system intended for patients with muscular dystrophy symptoms. They used hydraulic bilateral servo actuator (HBSA).

There can also be distinguished cable-driven constructions. One of them is MACARM [12]. It is a cable robot dedicated for neurorehabilitation. The robot is comprised of an array of 8 motors mounted at the corners of a cubic support frame. The end-effector has 6 DOF. This type of multi-axis robots is difficult to control and their ROM is limited.

Another type of cable-driven robots are anthropomorphic exoskeletons. The use of cable-driven technology to drive orthosis framework permit on safe space and the construction is light. However, the good lead of cables is difficult to attend and only few constructions are used that employ such a technology. A good example could be an ABLE robot [13]. It is a 4 axes exoskeleton with cable force transmission. It works by the new technology, which makes it possible to transmit force by cables and allows for highly compact arrangement. It is driven by a patented screw-and-cable system SCS [14].

Another example of a similar robot can be the construction patented by Univ et al [15]. The cable-driven exoskeleton is attached to the subject's 
limb. The exoskeleton has couplings between two or more joints of a limb of the subject. The joint can be adjusted to accommodate different limb sizes.

V. Zemlyakov and P. McDonough patented a robot and a method of rehabilitation used for whole arm rehabilitation: shoulder, elbow and wrist joints [16]. The exercises can be loaded on each joint simultaneously in every anatomical direction. Selected biomechanical information can be retrieved and stored in databases.

The most advanced are the robots driven by electric motors. This is because the electrically-driven motors are easy to use in exoskeleton structure (one can place motors in robot joints), and beside that, electric actuators are also relatively easy to control. There have already been developed many types of arm rehabilitation robots driven by electric motors. These constructions differ from each other, but they have one common feature - an exoskeleton structure. These apparatuses are at least capable of shoulder and wrist rehabilitation. One of such interesting structures was invented by Han Jungsoo et al [17]. They proposed a robot with high range of motion. The inventors achieve it by reducing volume of the actuators. The robot is used for shoulder and elbow rehabilitation. It is characterized by compact and elegant structure.

LExos is a new force-feedback arm exoskeleton [18]. Like other advanced robots, it can work in haptic interactions in Virtual Environments. The robot is used for shoulder and elbow rehabilitation and has 4 DOF actuated joints.

Carignan et al. developed a special anthropomorphic exoskeleton with a modular approach to control [19]. The construction has scapula and glenohumeral joints. In the grip handle there is a force sensor. Controller can operate in both impedance and admittance mode.

IntelliArm is one of the most advanced robots [20, 21]. The robot has $10 \mathrm{DOF}$, including hand opening and closing mechanism. It is used for shoulder, elbow and wrist rehabilitation. What is interesting, the robot is able to drive in four glenohumeral movements including flexion/extension, integral/external rotation, abduction/adduction and vertical displacement of the glenohumeral joint. The robot has passive DOFs in anterior/posterior and medial/laterial displament of glenohumeral joints. Forearm can be driven in flexion/extension and supination/pronation position.

And finally, one of the most advanced families of that kind of robots has been developed by scientists mainly related to the ETH Zurich. They are known by a common name - ARMin. The motor/gear combination is backdriveable, which is ensured by harmonic drive modules. A patient's arm is placed inside an orthotic shell. The first ARMin was presented more than 5 years ago. ARMin I has 6 degrees of freedom [22, 23]. The most recent ARMin III has 4 DOF (shoulder and elbow joints), but the wrist module can be also attached to the construction. The scientist from ARMin project 
proposed many types of advanced force-control algorithms. ARMin III has symmetrical structure. Because of that, it can be used on both sides - left and right arm of the subject [24].

There are also other interesting constructions of robots, which are similar to the presented ones. The above-mentioned systems are, however, probably the most advanced assistive-robots used for active arm rehabilitation.

\section{Device description}

The ARR robot consists of a base and active orthosis to which the upper extremity of the patient is fastened. The orthosis lies on the $X Y Z$ gantry system, which is fixed to the one of positioning axes of the base. $X Y Z$ gantry system moves actively during rehabilitation. The robot can be easily transported through a standard door (Fig. 1).

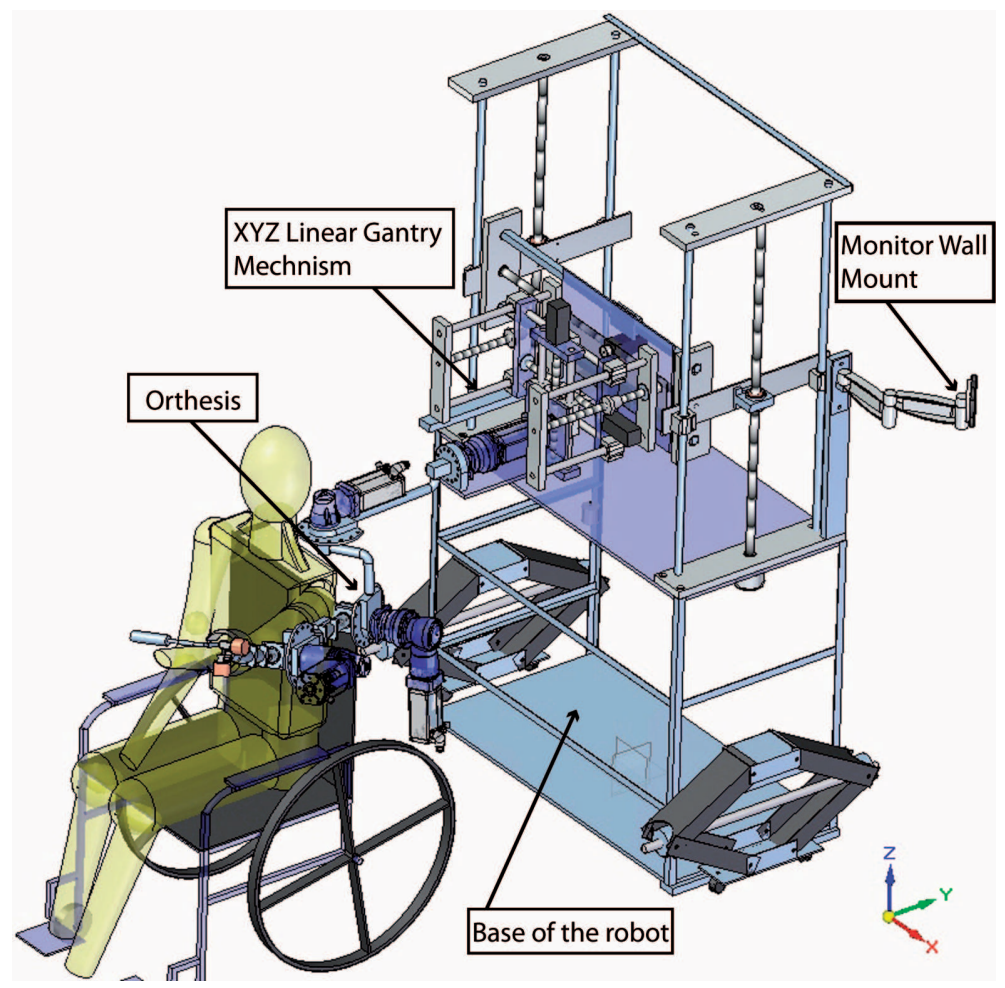

Fig. 1. General mechanical structure of ARR

The orthosis of the robot has a symmetric structure, and the robot can be used for both - right and left hand rehabilitation. It has wide ROM and can be used, for example, in rehabilitation of the patient in a sitting posture, as well as in standing position of the patient who is up to 2-meter tall. 
One of the main innovations in the constructed system will rely on using myosignals in active rehabilitation. Using myosignals provides possibilities of estimating forces and fatigue produced by the muscles. This will be possible by applying EMG apparatus and algorithm based on signal processing with some elements of artificial intelligence [25].

Another important part of the system is a subsystem of virtual environment. Movement parameters of an arm will be transmitted to the program environment. This information will be used to generate motion of virtual hand in Activity of Daily Living program.

\subsection{Base of the robot}

The base stands on supports, which makes the whole robot stable while performing rehabilitation. Special scissor jacks with wheels are connected to the base. By using it, the robot can be easily lifted and transported to another place. On the base, there are located most of critical devices of the system. There are low-level controllers of motors, a desktop computer, which is a main controller of the system, power supply adaptors, acquisition devices, EMG apparatus, screw terminals, fuses, capacitors and other devices. On the base, there are placed $X Z$ positioning axis, which adapt the orthosis to the anthropometric parameters of the patient's body.

\subsection{XYZ linear gantry system}

The orthosis is attached to an XYZ linear gantry system. This solution have been used in order to retain large ROM and sustain comfort to the patient. The centre of shoulder rotation changes during motion. Without applied linear actuators, an arm could move in the orthosis cuffs. The describing gantry system moves in three mutually orthogonal planes. The driving elements contain ballscrews, driven by stepper motors. Such actuators provide low friction and lower noise emissions.

\subsection{Orthosis}

The active orthosis is a construction with a rotary joints to which upper extremity of the patient is linked during rehabilitation.

The modular approach to the orthosis construction makes it possible to easily exchange old components for unused ones or add details of a new type. A good example of describing modularity could be glenohumeral joints, whose parts could be quickly replaced for the new parts with new geometry. In default joints, the configuration presented in Fig. 3 will be used, but 


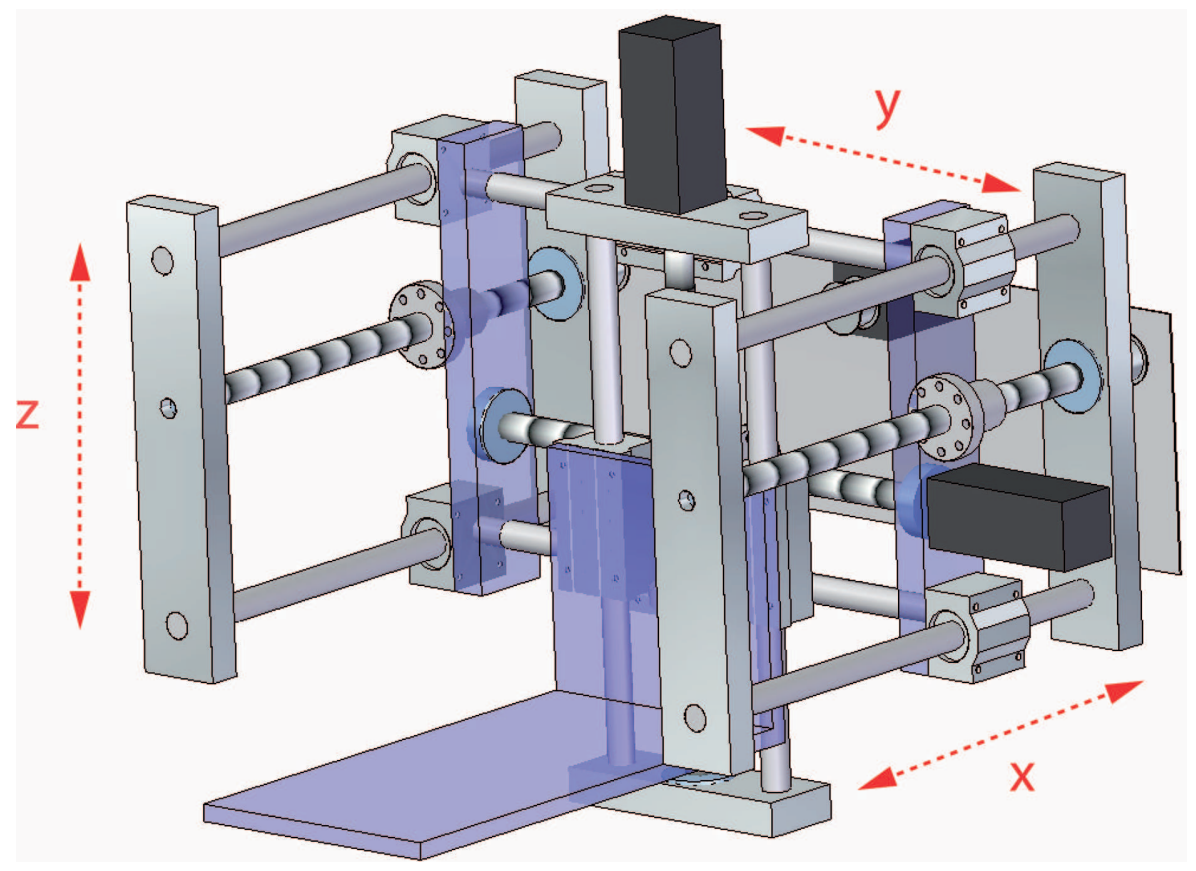

Fig. 2. The $X Y Z$ linear gantry mechanism

experiments will be also made on the joints similar to those presented in [26] patent.

The orthosis consists of upper and lower part. These parts are constructed of ballscrews and long nuts. The length of the parts can be automatically adjusted by DC motors with gears, according to the data of the patient stored in memory of the controller. The high ratio of the gear assures that the system is irreversible (rotation is locked). However, the second end of the lower nut is free. Two electromagnets are used to block its rotation. It can be switched on/off depending on the desired configuration (pronation/supination - on/off).

The orthosis has 4 active DOF. Three of them are responsible for glenohumeral joints movement, what with aforementioned linear joints gives 6 active DOF on shoulder girdle. One motor is responsible for flexion/extension of the elbow joint. The orthosis has also 3 DOF in wrist articulation. Precise encoders record the movement of a wrist, which is then transmitted to the virtual environment system.

A patient's arm is attached to a device via the cuffs covered with soft materials. The lower cuff is tightly connected to the forearm, while the upper cuff gives much more freedom to the upper arm. Special EMG apparatus will be situated inside the cuffs. 


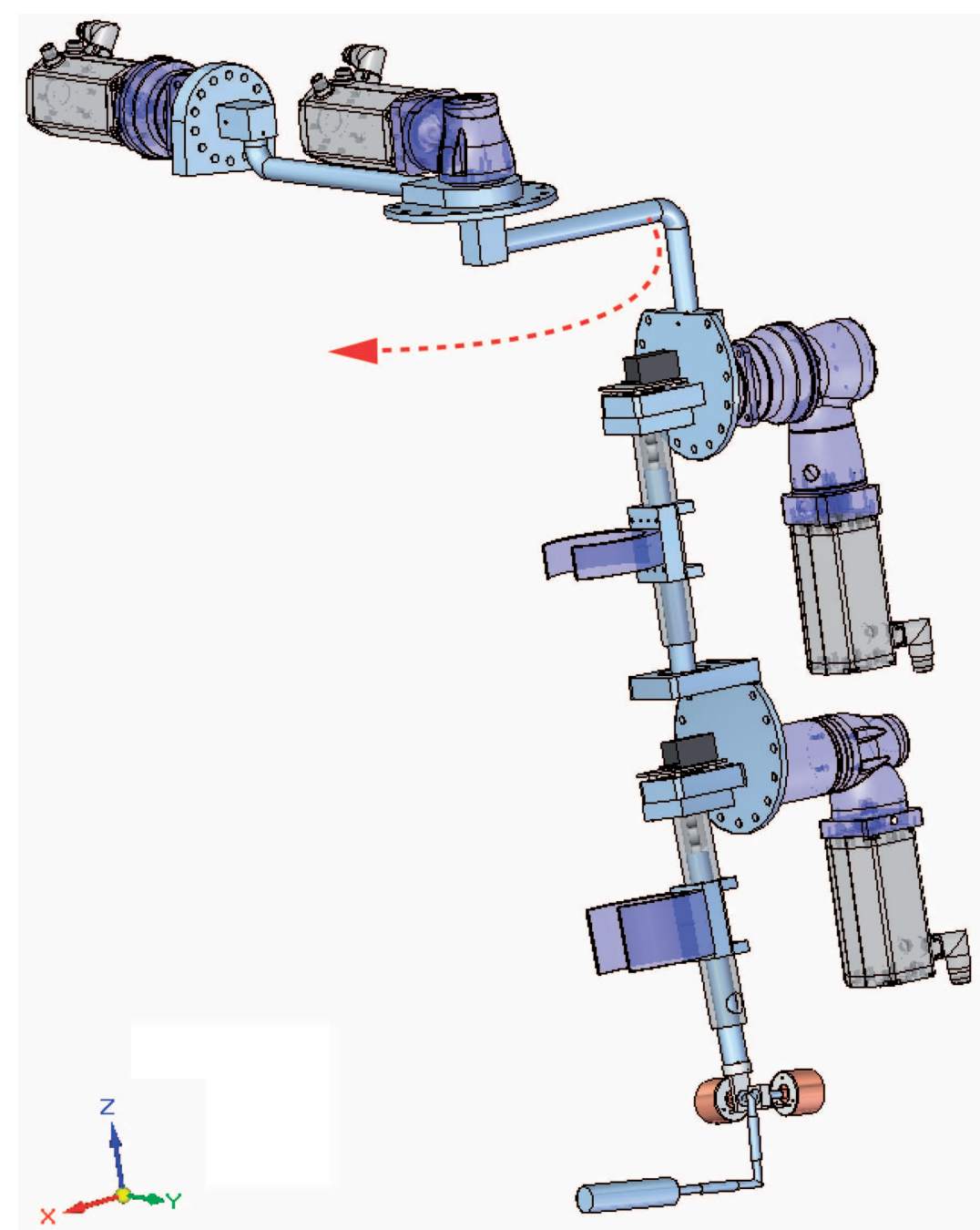

Fig. 3. Mechanical structure of the orthosis. In order to change rehabilitation on the other hand, the controlling system rotates the second rotary joint by 180 degree, as is indicated by a red arrow

The orthosis has also simple mechanical limiters of motion. These are discs with threaded holes into which bolts can be screwed. It is the last line of the multi-modular mechanical safety system.

Main drives of an orthosis consist of BLDC (Brushless DC) motors, planetary gears and safety couplings. The torque of drives can be easily controlled. The motors with gears are backdriveable. This allows one to control robots in advanced impedance/admittance mode and give patients possibilities to move joints by themselves, while the robot will support the movements. 


\subsection{Low-level controllers}

Robot consists of several low level controllers, which are used for transmitting information from the high-level controller (programs situated on desktop computer) to robot actuators, as well as from sensors to the high-level controller. These electronic controllers are connected to a computer through Data Acquisition Cards, PCI-CAN card and through microprocessors using UART-USB protocol.

Biological sensors transmit information to a high-level controller through a microprocessor $(\mu \mathrm{P})$ with UART/USART protocol. Also encoders are connected to a computer through another low-level controller with architecture based on $\mu \mathrm{P}$ with UART protocol (Fig. 4).

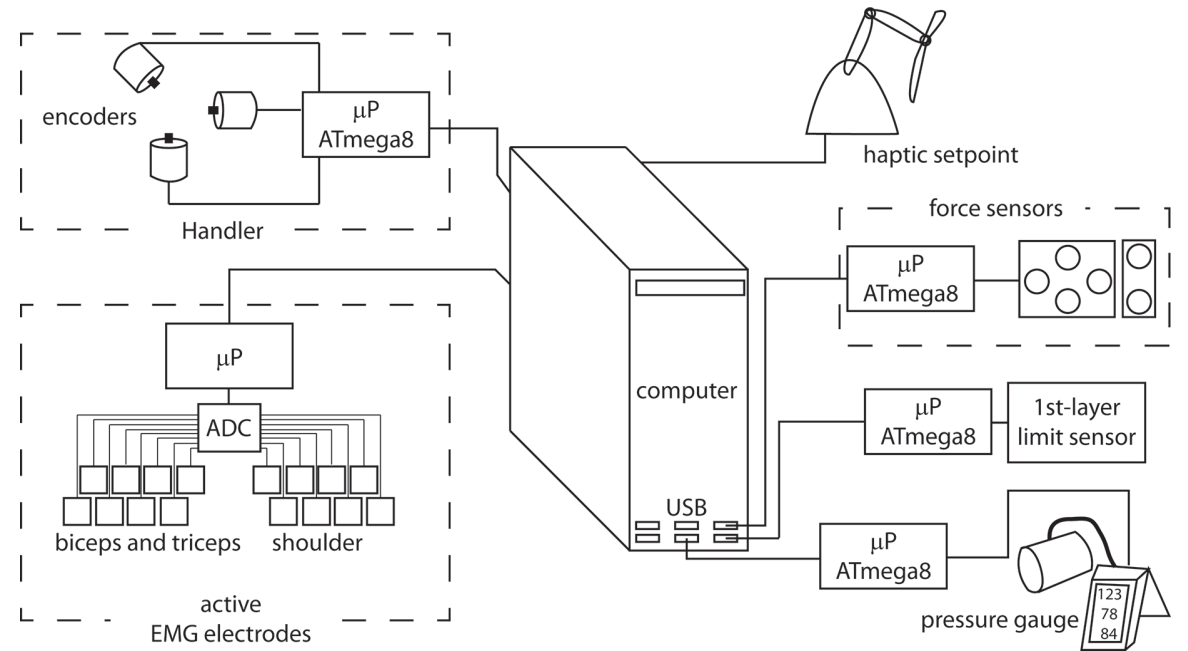

Fig. 4. General scheme of low-level controllers, which communicate with high-level controller by USB

DC motors, which are responsible for positioning process before rehabilitation, are linked to computer through USB DAQ module (Fig. 5). Motors, which move actively during rehabilitation, are controlled by high-level controller through CAN protocol with PCI-CAN card and through digital and analogue I/O cards.

\subsection{Motors}

There are 11 motors installed in the robot. Two DC motors are situated in the basis of the robot. These motors are used for adjusting the position of the orthosis to patient height and width of his shoulder before rehabilitation. This is done by changing the position of the $X Z$ main linear gantry system. Two smaller DC motors with worm gears are used to change the length of 

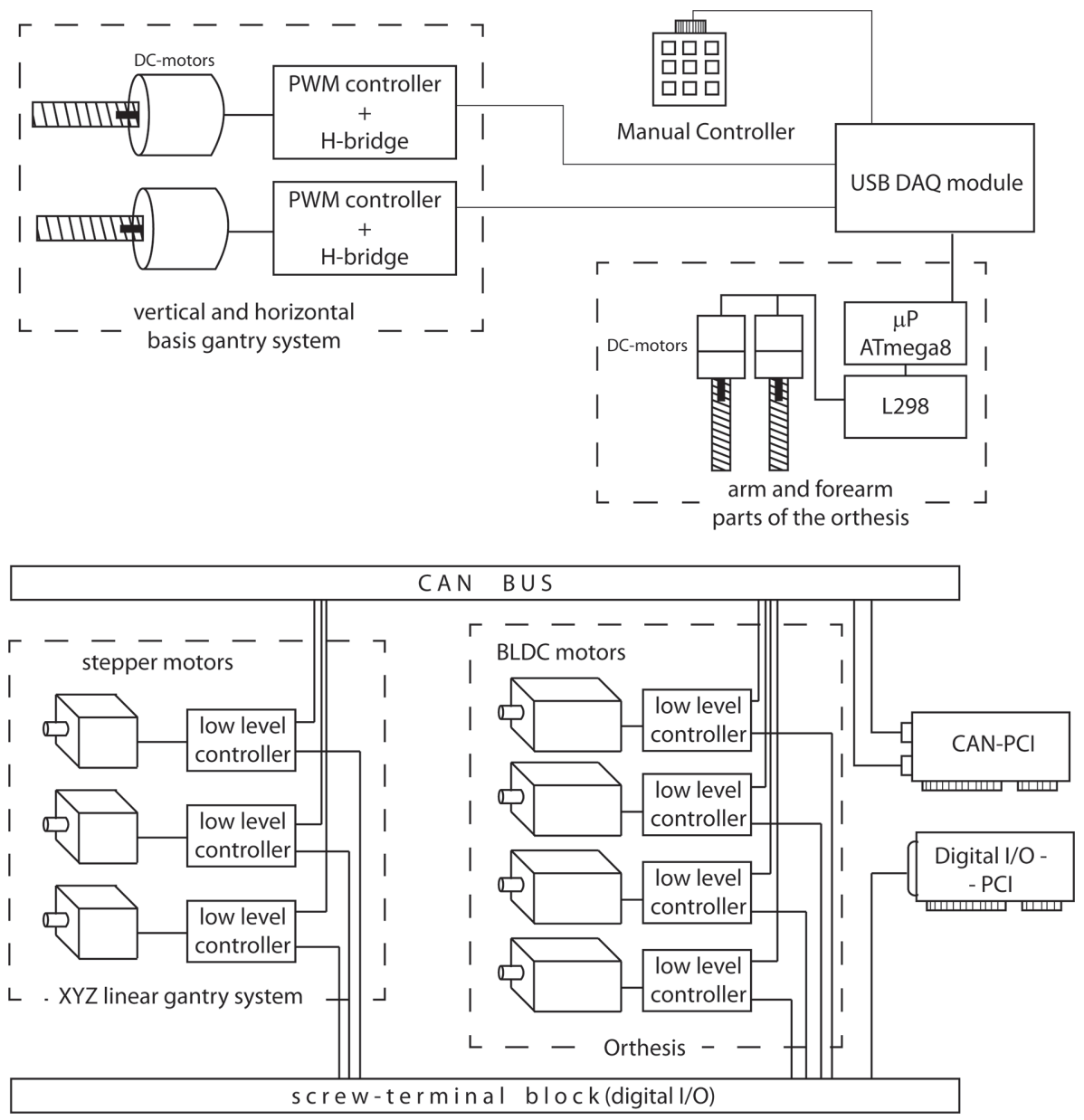

Fig. 5. Structure of low-level controllers of BLDC and stepper motors

the arm and forearm parts of the orthosis. This is important for providing non-backdriveability of the transmissions of this part of orthosis during rehabilitation. Worm gears cause that arm and forearm parts of the orthosis do not move during rehabilitation, even when motors are turned off after length adjustment process.

Seven motors move actively during rehabilitation. Three of them are responsible for positioning the $X Y Z$ gantry system Stepper motors without gears are used there. These motors are necessary, because pivot point of a shoulder translates according to the position of an upper extremity. High level controller steers these motors, even during the learning process.

The most important are aforementioned motors, which drive the orthesis. BLDC motors are used there. The force is transmitted through planetary gears. The power and type of these motors are the same, only gear ratios are 
different. This approach let the researchers test different algorithms for forceposition control, and check how transmission affects this type of control.

\subsection{Sensors}

The robot have many position sensors (mainly encoders), which are indispensable for proper control. The robot is prepared for implementation of advanced biofeedback algorithms. Because of this, the system also has biological sensors. The most important of them are EMG sensors. Based on the signal recorded by EMG sensors, one can estimate which motor muscle unit is activated, with what force and, moreover, what is the fatigue of the muscles. There are two groups of EMG sensors, arranged in a matrix, which are situated on arm and shoulder during rehabilitation.

There are also pulse and blood pressure sensors, which help to estimate tiredness of the patient. The pulse sensor has also an emergency function. In the future, additional information about muscle fatigue will be also recorded from Galvanic Skin Resistance (GSR) sensors. The biofeedback sensors will help to develop an algorithm, which will predict the progress of rehabilitation.

\section{Safety Systems}

The robot has multi-level safety system. Safety system guarantee harmless exercises and experiments on the robot prototype. A good security system also ensures safety of patients. First line of security are subprograms located in high-level controller, which instantly provide an assessment of functioning of other subprograms, for example whether subprograms correctly interpret the limits of ROM or whether they produce correct checksum. There is also a special subprogram, which will interpret the pulse and blood pressure of patients. Rehabilitation stops when pulse rate is below or above an acceptable threshold. On the lower level, there act programs in $(\mu \mathrm{Ps})$, which are independent from the programs located on the computer. These programs check correctness of incoming and outgoing data. There are also watchdogs, which verify whether uPs operate properly.

On the lower level, there are also electrical sensors. Especially important is stopping the robot in the case of exceeding the allowable range of movement by machine components, otherwise robot could break patient's arm. A good example of that type of safety sensors can be special double-layer end position sensors (Fig. 6). In the case of exceeding the allowable range, at the beginning the first sensor would send a signal to stop the robot and if it would not bring an effect, the second sensor would turn off the motor power. In very rare cases of a damaged relay, the robot would be stopped 
by mechanical limiters (Fig. 3) and the motors power would be cut off by overcurrent protection.

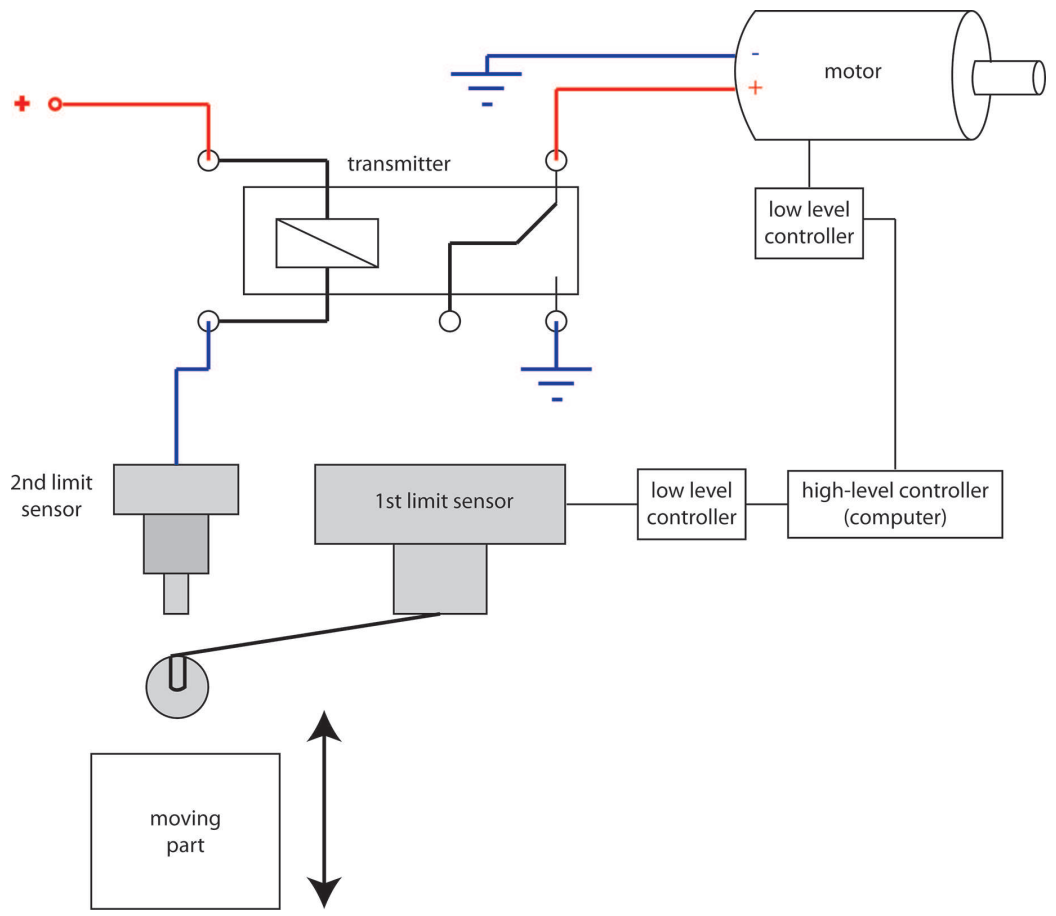

Fig. 6. Two-layer position sensor switch

\section{Kinematic structure}

The aim of the design is to reflect kinematics of human arm structure. The arm of a human body has 7 DOF [27]. In the exoskeleton structure, there can be distinguished, similarly to the human arm, the shoulder girdle, the upper arm part, forearm and the grip on hand (Fig. 7). In the construction, there is no rotary joint reflecting scapula joint of the human body. Instead of it, there is a 3D planar gate. This approach has three main advantages. The planar gate has much more ROM, it can compensate shoulder displacements better than one scapula joint and with a special algorithm it will be able to react even to torso movements. To the planar gate, there are connected 3DOF revolute joints, corresponding to spherical glenohumeral $(\mathrm{GH})$ joints of the human body. The centre of rotation is located at the centre of a humerus head. GH1 joint 1 replicates adduction-abduction movements, GH2 correspond to rotation and the last $\mathrm{GH} 3$ replicates flexion-extension movements.

The fourth active revolute joint is situated in an elbow position (EL1). It is responsible for flexion-extension of the forearm. The robot has also 3 


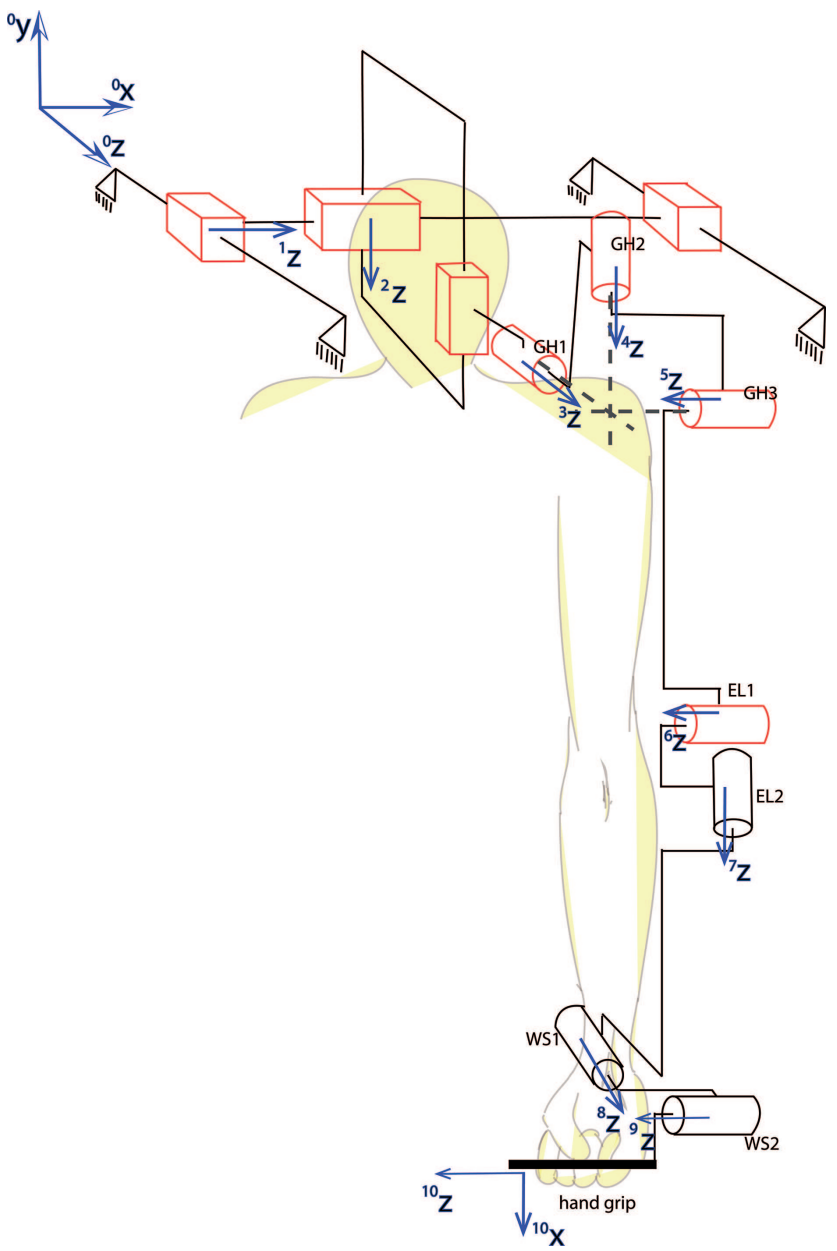

Fig. 7. Kinematic structure of the robot (without adjusting joints). Active joints are marked red

passive joints, corresponding to pronation/supination of the forearm (EL2), adduction-abduction (WS1) and flexion-extension (WS2) of the hand. Patient's hand lies on the sensored hand grip (Table 1).

\section{Procedure of rehabilitation}

The procedure of rehabilitation differs whatever rehabilitation take place for the first time, or whether it is another session with the same patient. When a rehabilitation process takes place for the first time, a therapist has to measure anthropological parameters of the patient and save them in the database. There will be an intuitive, ergonomic Graphic User Interface (GUI), so that a trained therapist should not have any problems with using the program. When patient's parameters are already saved in the database, the 
ARTUR GMEREK

Table 1.

DH Table for 10-DOF orthesis. Variables are indicated with a star $(*)$.

This DH table refers to Fig. 7

\begin{tabular}{|c|c|c|c|c|}
\hline $\mathbf{i}$ & $\mathbf{a}_{\mathbf{i}}$ & $\alpha_{\mathbf{i}}$ & $\mathbf{d}_{\mathbf{i}}$ & $\theta_{\mathbf{i}}$ \\
\hline 1 & 0 & $\frac{\pi}{2}$ & $d_{1}^{*}$ & $\frac{\pi}{2}$ \\
\hline 2 & 0 & $-\frac{\pi}{2}$ & $d_{2}^{*}$ & $\frac{\pi}{2}$ \\
\hline 3 & 0 & $\frac{\pi}{2}$ & $d_{3}^{*}$ & $-\frac{\pi}{2}$ \\
\hline 3.5 & $a_{3.5}$ & 0 & 0 & $\theta_{3.5}^{*}+\frac{\pi}{2}$ \\
\hline 4 & 0 & $\frac{\pi}{2}$ & $d_{4}$ & $-\frac{\pi}{2}$ \\
\hline 4.5 & $a_{4.5}$ & 0 & 0 & $\theta_{4.5}^{*}$ \\
\hline 5 & 0 & $-\frac{\pi}{2}$ & $d_{5}$ & $\frac{\pi}{2}$ \\
\hline 6 & $a_{6}$ & 0 & 0 & $\theta_{6}^{*}-\frac{\pi}{2}$ \\
\hline 7 & 0 & $\frac{\pi}{2}$ & 0 & $\theta_{7}^{*}+\frac{\pi}{2}$ \\
\hline 7.5 & 0 & 0 & $d_{7.5}$ & $\theta_{7.5}^{*}+\pi$ \\
\hline 8 & $a_{8}$ & $\frac{\pi}{2}$ & $d_{8}$ & $-\frac{\pi}{2}$ \\
\hline 9 & 0 & $\frac{\pi}{2}$ & $d_{9}$ & $\theta_{9}^{*}+\frac{\pi}{2}$ \\
\hline 10 & $a_{10}$ & 0 & $d_{10}$ & $\theta_{10}^{*}$ \\
\hline
\end{tabular}

therapist can load the data in order to start rehabilitation. After that, the patient can sit down or stand back to the robot. The therapist moves gantry system together with connected orthosis. After situating it near the patient's arm, the therapist changes the length of arm and forearm parts and connects patient's upper extremity to the orthosis. After that, the therapist can program the robot, load some previously recorded exercises, or put the robot in a quasichaotic movement. The orthosis-arm arrangement is moving slowly through a defined trajectory. In the end, after training, the arm can be detached from the orthosis. All needed data are saved in the database. After rehabilitation, special algorithm can be also started which, on the basis of the recorded EMG signal, estimates the progress of rehabilitation and updates rehabilitation's parameters (dynamic of motion, period of exercise, range of motion) useful for future therapeutic sessions. 


\section{Discussion}

Many aspects of the presented robot can be subject to discussion. The most important is the kinematic structure. Other designers have usually used a different configuration with a scapula joint. Nevertheless, the author believes that the selected solution of 3-DOF linear guideways is highly suitable, because it widely increases ROM of the orthosis, and lets the robot to react even to torso movements. This denouement facilitates manoeuvring, maximizes the variety of possible types of rehabilitation, and makes it more comfortable for the patient.

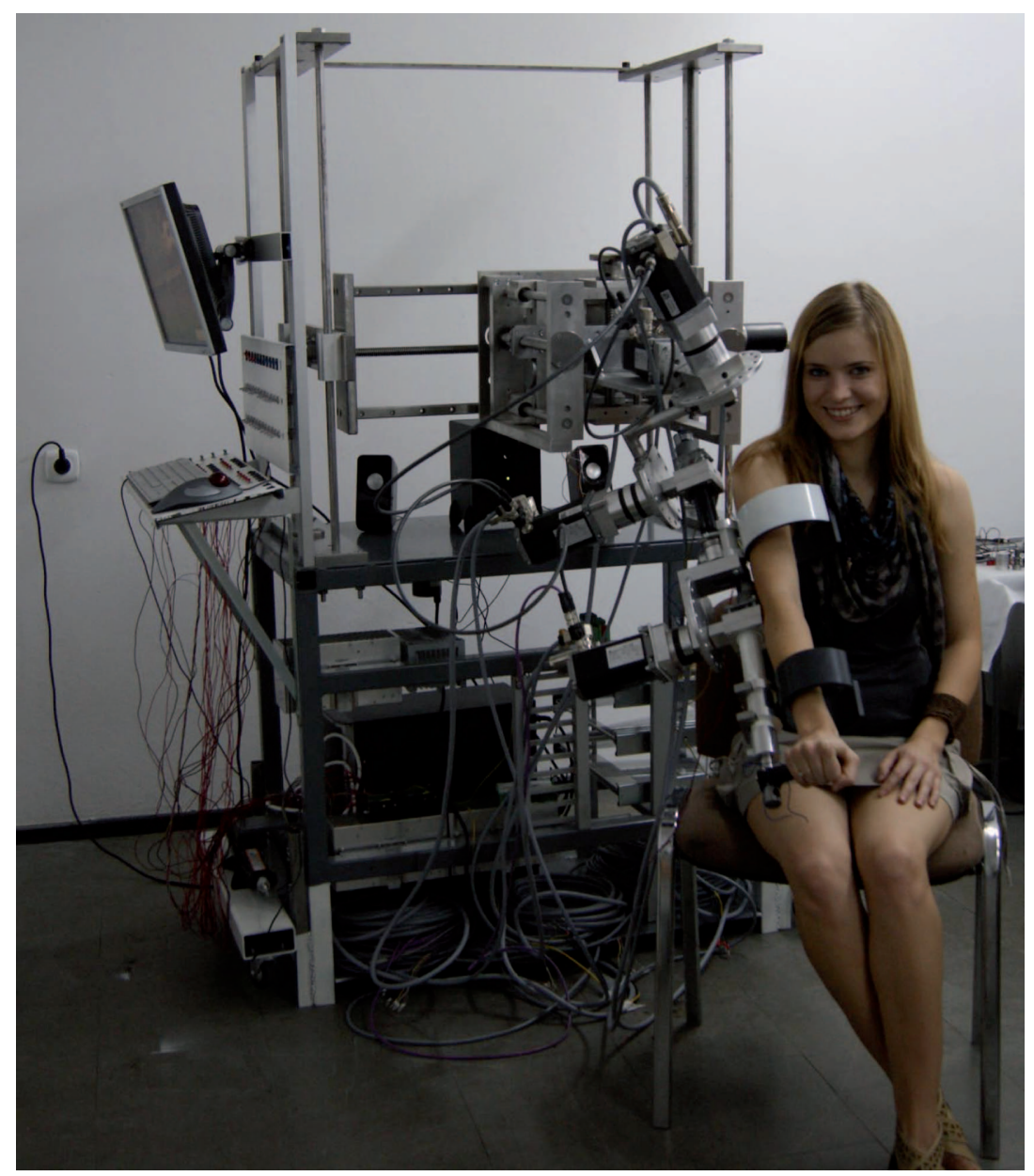

Fig. 8. Described rehabilitation robot 


\section{Conclusions and Future Work}

In this paper, the mechanical structure of a novel rehabilitation robot has been presented. The robot has a semi-exoskeleton configuration and unique kinematic structure. The design was optimized for potential types of rehabilitation exercises and safety mechanism. The construction is also optimized with respect to costs. Design and creation of the mechanical structure is the first step on the way to finish this project. The final effect, probably to be achieved in the next year, will be a complete upper extremity rehabilitation system. The system will work in position, or impedance/admittance mode.

\section{Acknowledgment}

This work is financially supported by the Ministry of Science and Higher Education of Poland (Grant No. N N514 469339).

I am grateful for valuable insight into this work and advice to Prof. Edward Jezierski from Institute of Automatic Control of the Technical University of Lodz.

Manuscript received by Editorial Board, November 15, 2012; final version, September 29, 2013.

\section{REFERENCES}

[1] Parasuraman S., Yee K.C., Oyong A.: "Human upper limb and arm kinematics for robot based rehabilitation," in Proc. IEEE/ASME Int. Conf. Advanced Intelligent Mechatronics AIM 2009, 2009, pp. 845-850.

[2] Hogan N., Krebs H., Charnnarong J., Srikrishna P., Sharon A.: "MIT-MANUS: a workstation for manual therapy and training." in IEEE International Workshop on Robot and Human Communication, 1992.

[3] Formica D., Zollo L., Guglielmelli E.: "Torque-dependent compliance control in the joint space of an operational robotic machine for motor therapy," in Proc. 9th Int. Conf. Rehabilitation Robotics ICORR 2005, 2005, pp. 341-344.

[4] Reinkensmeyer D.J., Wolbrecht E., Bobrow J.: "A computational model of human-robot load sharing during robot-assisted arm movement training after stroke," in Proc. 29th Annual Int. Conf. of the IEEE Engineering in Medicine and Biology Society EMBS 2007, 2007, pp. 4019-4023.

[5] Wolbrecht E.T., Leavitt J., Reinkensmeyer D.J., Bobrow J.E.: "Control of a pneumatic orthosis for upper extremity stroke rehabilitation," in Proc. 28th Annual Int. Conf. of the IEEE Engineering in Medicine and Biology Society EMBS '06, 2006, pp. 2687-2693.

[6] Jackson A.E., Makower S.G., Culmer P.R., Holt R.J., Cozens J.A., Levesley M.C., Bhakta B.B.: "Acceptability of robot assisted active arm exercise as part of rehabilitation after stroke," in Proc. IEEE Int. Conf. Rehabilitation Robotics ICORR 2009, 2009, pp. 103-108.

[7] Mistry M., Mohajerian P., Schaal S.: "Arm movement experiments with joint space force fields using an exoskeleton robot," in Proc. 9th Int. Conf. Rehabilitation Robotics ICORR 2005, 2005, pp. 408-413. 
[8] Kousidou S., Tsagarakis N.G., Smith C., Caldwell D.G.: "Task-orientated biofeedback system for the rehabilitation of the upper limb," in Proc. IEEE 10th Int. Conf. Rehabilitation Robotics ICORR 2007, 2007, pp. 376-384.

[9] Balasubramanian S., Wei R., Perez M., Shepard B., Koeneman E., Koeneman J., He J.: "RUPERT: An exoskeleton robot for assisting rehabilitation of arm functions," in Proc. Virtual Rehabilitation, 2008, pp. 163-167.

[10] Sugar T.G., He J., Koeneman E.J., Koeneman J.B., Herman R., Huang H., Schultz R.S., Herring D.E., Wanberg J., Balasubramanian S., Swenson P., Ward J.A.: "Design and control of RUPERT: A device for robotic upper extremity repetitive therapy," IEEE Transactions on Neural Systems and Rehabilitation Engineering, vol. 15, no. 3, pp. 336-346, 2007.

[11] Umemura A., Saito Y., Fujisaki K.: "A study on powerassisted rehabilitation robot arms operated by patient with upper limb disabilities," in Proc. IEEE Int. Conf. Rehabilitation Robotics ICORR 2009, 2009, pp. 451-456.

[12] Beer R., Mayhew D., Bredfeldt C., Bachrach B.: "Technical evaluation of the MACARM: A cable robot for upper limb neurorehabilitation,” in Proc. 2nd IEEE RAS \& EMBS Int. Conf. Biomedical Robotics and Biomechatronics BioRob 2008, VOL. 1, NO. 11, NOVEMBER 2002 9, pp. 942-947.

[13] Jarrasse N., Robertson J., Garrec P., Paik J., Pasqui V., Perrot Y., Roby-Brami A., Wang D., Morel G.: "Design and acceptability assessment of a new reversible orthosis," in Proc. IEEE/RSJ Int. Conf. Intelligent Robots and Systems IROS 2008, 2008, pp. 1933-1939.

[14] Philippe G., Gif-sur Y.: "Screw and nut transmission and cable attached to the screw," U.S. Patent US 7,073,406 B2, 2006.

[15] Univ K., Scott S.H., Brown I.E., Ball S.J.: "Robotic exoskeleton for limb movement," Worldwide Patent WO2 008131563 (A1), 2008.

[16] Zemlyakov V., McDonough P.: "Upper extremity exoskeleton structure and method," U.S. Patent US2 003115954 (A1), 2003.

[17] Jungsoo H., Changsoo H., Hyeyoen J., Jaeho J., Youngsu L., Sungjoon H.: "Wearable robotic system for rehabilitation training of the upper limbs," Worldwide Patent WO2 010071252 (A1), 2010.

[18] Frisoli A., Borelli L., Montagner A., Marcheschi S., Procopio C., Salsedo F., Bergamasco M., Carboncini M.C., Tolaini M., Rossi B.: "Arm rehabilitation with a robotic exoskeleleton in virtual reality," in Proc. IEEE 10th Int. Conf. Rehabilitation Robotics ICORR 2007, 2007, pp. 631-642.

[19] Carignan C., Tang J., Roderick S., Naylor M.: "A configuration-space approach to controlling a rehabilitation arm exoskeleton," in Proc. IEEE 10th Int. Conf. Rehabilitation Robotics ICORR 2007, 2007, pp. 179-187.

[20] Ren Y., Park H.-S., Zhang L.-Q.: "Developing a whole-arm exoskeleton robot with hand opening and closing mechanism for upper limb stroke rehabilitation,” in Proc. IEEE Int. Conf. Rehabilitation Robotics ICORR 2009, 2009, pp. 761-765.

[21] Li-Qun Z., Hyung-Soon P., Yupeng R.: "Robotic rehabilitation apparatus and method," U.S. Patent US2 010016766 (A1), 2010.

[22] Nef T., Mihelj M., Colombo G., Riener R.: "ARMin - robot for rehabilitation of the upper extremities," in Proc. IEEE Int. Conf. Robotics and Automation ICRA 2006, 2006, pp. 3152 3157.

[23] Nef T., Riener R.: "ARMin - design of a novel arm rehabilitation robot," in Proc. 9th Int. Conf. Rehabilitation Robotics ICORR 2005, 2005, pp. 57-60.

[24] Guidali M., Schmiedeskamp M., Klamroth V., Riener R.: "Assessment and training of synergies with an arm rehabilitation robot," in Proc. IEEE Int. Conf. Rehabilitation Robotics ICORR 2009, 2009, pp. 772-776.

[25] Gmerek A.: "High-level controller for an arm rehabilitation robot - positioning algorithms with respect to EMG data," in Proc. IEEE Int. Conf. MMAR 2011, 2011, pp. 182-187. 
[26] Chang Soo H., Jung Soo H., Tae Sick K., Hye Youn J., Jae Ho J., "Development of wearable robot mechanism for 3 DOF shoulder joint behavior,” Korean Patent KR20 090036177 (A), 2009.

[27] Parasuraman S., Oyong A.W., Ganapathy V.: "Development of robot assisted stroke rehabilitation system of human upper limb," in Proc. IEEE Int. Conf. Automation Science and Engineering CASE 2009, 2009, pp. 256-261.

\section{Projekt mechaniczny i architektura systemu sterowania semi-egzoszkieletalnego robota przeznaczonego do rehabilitacji kończyny górnej}

\section{Streszczenie}

Artykuł opisuje mechanikę oraz część sprzętową systemu sterowania robota przeznaczonego do rehabilitacji kończyny górnej. Robot został nazwany Arm Rehabilitation Robot - ARR i charakteryzuje się strukturą semi-egzoszkieletalną. Oznacza to, że część aktywna przylega do ciała pacjenta, a jego struktura kinematyczna przypomina kończynę górną, ale waga robota nie obciąża pacjenta. Urządzenie może być używane do rehabilitacji całej kończyny górnej, ale aktywne stopnie swobody znajdują się w stawach barkowo-obojczykowym oraz łokciowym. Robot jest napędzany silnikami elektrycznymi. 